\title{
Time-dependent treatment of cosmic-ray spectral steepening due to turbulence driving
}

\author{
Martin Pohl ${ }^{a, b, *}$ \\ ${ }^{a}$ DESY, DE-15738 Zeuthen, Germany \\ ${ }^{b}$ Institute of Physics and Astronomy, University of Potsdam, DE-14476 Potsdam, Germany \\ E-mail: marpohl@uni-potsdam.de
}

\begin{abstract}
Cosmic-ray acceleration at non-relativistic shocks relies on scattering by turbulence that the cosmic rays drive upstream of the shock. We explore the rate of energy transfer from cosmic rays to non-resonant Bell modes and the spectral softening it implies. Accounting for the finite time available for turbulence driving yields a much smaller spectral impact than found earlier with steady-state considerations. Generally, for diffusion scaling with the Bohm rate by a factor $\eta$, the change in spectral index is at most $\eta$ divided by the Alfvénic Mach number of the thermal sub-shock. For small $M_{A}$ it is well below this limit. Only for very fast shocks and very efficient cosmic-ray acceleration the change in spectral index may reach 0.1 . For standard SNR parameters it is negligible.
\end{abstract}

$37^{\text {th }}$ International Cosmic Ray Conference (ICRC 2021)

July 12 th - 23rd, 2021

Online - Berlin, Germany

\footnotetext{
*Presenter
} 


\section{Introduction}

[1] studied cosmic-ray acceleration at nonrelativistic shocks using a tensor expansion of the Vlasov equation, and they found a steepening of the cosmic-ray spectrum arising from energy transfer from the cosmic rays to turbulence in the precursor of the shocks. Assuming the turbulence in question is nonresonant, the so-called Bell mode [2], and taking an estimate for its energy density at the saturation level,

$$
U_{\delta B} \approx \frac{v_{\mathrm{sh}}}{2 c} U_{\mathrm{cr}},
$$

where $v_{\text {sh }}$ denotes the shock speed and $U_{\text {cr }}$ the energy density in cosmic rays immediately upstream of the shock, they find a spectral steepening by

$$
\left.\Delta s\right|_{\text {Bell }} \approx \frac{4}{\epsilon} \frac{U_{\delta B}}{U_{\mathrm{cr}}} \approx \frac{2}{\epsilon} \frac{v_{\mathrm{sh}}}{c} .
$$

Here $\epsilon$ is the fraction of turbulent energy density that is in the form of magnetic field. For, e.g., Alfvén waves we have $\epsilon=0.5$.

What of the uncertainties in the estimate? Does turbulence driving perhaps impose termination of acceleration as opposed to a continuous spectral steepening? Which fraction of the observed post-shock magnetic energy density is actually carried by Bell's mode and not by provided by any other process operating at the shock [e.g. 5]? Do the waves at the shock fully sample the cosmic-ray energy loss or have the waves lost energy already in the upstream region? And is the analytically estimated saturation level really relevant? Is there enough time for Bell's mode to grow to very high amplitude in the precursor of a shock, before the plasma arrives at the shock and the driving of waves stops? For simplicity we consider cosmic rays with Lorentz factor $\Gamma_{\mathrm{cr}}$. The peak growth rate of the mode scales with the proton gyrofrequency, $\Omega_{\mathrm{p}}$, or with the ion plasma frequency, $\omega_{\mathrm{p}, \mathrm{p}}$, as

$$
\gamma_{\max } \simeq \Omega_{\mathrm{p}} \frac{v_{\mathrm{sh}} N_{\mathrm{cr}}}{2 v_{\mathrm{A}} N_{\mathrm{p}}}=\omega_{\mathrm{p}, \mathrm{p}} \frac{v_{\mathrm{sh}} N_{\mathrm{cr}}}{2 c N_{\mathrm{p}}},
$$

where $v_{\mathrm{A}}$ denotes the Alfvén speed and $N$ is the number density of cosmic rays (cr) or ambient protons (p).

\section{Time-dependent modeling of turbulence build-up}

We shall explore two independent ways to estimate the energy transfer from cosmic rays to turbulence. One will be a local consideration of turbulence driving in the precursor, the other one an integral assessment of the energy transfer in the entire precursor. For simplicity we shall assume that all ions in the cosmic rays and in the background plasma are protons, i.e. hydrogen nuclei. The non-resonant and broadband character of Bell's mode permits considering cosmic rays of a specific energy as proxy for particles in a wide energy band.

\subsection{Local estimate}

The growth rate of wave energy density is proportional to the wave energy in the unstable wave band,

$$
\dot{U} \simeq \int d k \gamma(k) \frac{B_{k}^{2}}{4 \pi \epsilon} \lesssim \gamma_{\max } \frac{(\delta B)^{2}}{4 \pi \epsilon},
$$


where again $\epsilon$ is the fraction of the wave energy that is carried by magnetic field, and $\delta B$ is the magnetic wave amplitude. Equation 4 indicates that most of the energy transfer arises when the wave amplitude is high, meaning near or at saturation. Inserting Eq. 3 gives

$$
\dot{U} \lesssim \frac{\omega_{\mathrm{p}, \mathrm{p}}}{\epsilon} \frac{N_{\mathrm{cr}}}{N_{\mathrm{p}}} \frac{v_{\mathrm{sh}}}{c} U_{\delta B}
$$

A gain in energy density of the waves is a loss in energy density of the driving cosmic rays. We can estimate the energy-loss time per cosmic-ray particle as

$$
\tau_{\text {loss }} \simeq \frac{U_{\mathrm{cr}}}{\dot{U}} \gtrsim \frac{2 \epsilon \Gamma_{\mathrm{cr}}}{\omega_{\mathrm{p}, \mathrm{p}}} \frac{U_{\mathrm{bulk}}}{U_{\delta B}} \frac{c^{3}}{v_{\mathrm{sh}}^{3}},
$$

which evidently is independent of the number density of cosmic rays.

We shall now compare the energy-loss time (Eq. 6) with the acceleration time assuming diffusive shock acceleration. Again expressing the diffusion coefficient of relativistic cosmic rays in Bohm units, $\kappa=\eta c r_{\mathrm{L}} / 3$, we rewrite equation (32) of Drury [4] as

$$
\tau_{\mathrm{acc}}=\frac{8 \kappa}{v_{\mathrm{sh}}^{2}}=\frac{8 \eta \Gamma_{\mathrm{cr}}}{3 \Omega_{\mathrm{p}}} \frac{c^{2}}{v_{\mathrm{sh}}^{2}} .
$$

[8] noted that for efficient magnetic-field amplification we can either write $\kappa$ with the far-upstream magnetic field, $B_{0}$, and $\eta<1$, or, alternatively, we can use the amplified field strength and $\eta \approx 4$. In this paper we shall do the latter. The spectral index is $s-1=\tau_{\text {acc }} / \tau_{\text {esc }}$. Energy losses by driving turbulence increase the effective acceleration time scale, leading to a softened spectrum

$$
\frac{d N}{d E} \approx \frac{\Delta N}{\Delta E} \simeq \frac{1-s}{1-\frac{\tau_{\text {acc }}}{\tau_{\text {loss,eff }}}} \frac{N}{E}
$$

The ratio of timescales in the denominator of eq. 8 must be less than unity, otherwise acceleration is impossible. For a small ratio of acceleration time and loss time the resulting change of spectral index can be written using the Alfvénic Mach number, $M_{\mathrm{A}}=c \Omega_{\mathrm{p}} / \omega_{\mathrm{p}, \mathrm{p}}$, that is calculated with the amplitude of the amplified field, i.e. without regard of direction,

$$
\Delta s \lesssim \frac{2(s-1) \eta M_{\mathrm{A}}}{3 \epsilon} \frac{U_{\delta B}}{U_{\text {bulk }}} .
$$

\subsection{Global assessment}

We shall now conduct a global assessment of the cosmic-ray energy loss incurred in the entire upstream region. The spatial profile of cosmic-ray density in the so-called precursor is dominated by the homogeneous solution to the spatial part of the transport equation,

$$
N_{\mathrm{cr}} \simeq N_{\mathrm{cr}, \mathrm{sh}} \exp \left(-\int_{r_{\mathrm{sh}}}^{r} \frac{d r^{\prime} v\left(r^{\prime}\right)}{\kappa\left(r^{\prime}\right)}\right)
$$

where $v(r)$ is the upstream flow speed measured in the shock rest frame.

The cosmic-ray density profile also determines the acceleration timescale, because it depends on the average separation of upstream cosmic rays from the shock, $\left\langle r-r_{\mathrm{sh}}\right\rangle$. In each acceleration 
cycle, cosmic rays enjoy a relative energy gain on the order of $v_{\mathrm{sh}} / c$ and need a few times $\left\langle r-r_{\mathrm{sh}}\right\rangle / c$ in time for it. Hence the acceleration time is a few times $\left\langle r-r_{\mathrm{sh}}\right\rangle / v_{\mathrm{sh}}$. If $v$ and $\kappa$ were constant, then

$$
\left\langle r-r_{\mathrm{sh}}\right\rangle=\frac{\kappa}{v}=\frac{\kappa}{v_{\mathrm{sh}}}
$$

and we recover the usual $\tau_{\mathrm{acc}} \propto \kappa / v_{\mathrm{sh}}^{2}$ [4]. If $v / \kappa$ would significantly decrease at $r-r_{\mathrm{sh}} \gg\left\langle r-r_{\mathrm{sh}}\right\rangle$, then we would arrive at approximately the same conclusion, except that there might be some (weak) escape toward the far upstream. If $v / \kappa$ would significantly decrease already close to the shock, then $\left\langle r-r_{\mathrm{sh}}\right\rangle$ would be very large and may in fact become unbound, in which case many of the freshly accelerated cosmic rays escape to the far upstream, and the cosmic-ray spectrum would be very steep [3]. The rapid increase of volume with increasing $r$ would contribute to the cut-off in the spectrum of confined particles [7].

We shall now explicitly consider variations in the flow speed and hence write eq. 3 with $v(r)$ instead of $v_{\text {sh }}$. Together with eq. 4 we can integrate the energy transfer rate over the entire precursor, which for a plane-parallel shock means integration over $r$,

$$
\dot{E}_{\mathrm{tot}} \lesssim \frac{N_{\mathrm{cr}, \mathrm{sh}}}{8 \pi \epsilon c} \int_{r_{\mathrm{sh}}}^{\infty} d r \frac{\omega_{\mathrm{pp}} v(r)(\delta B(r))^{2}}{N_{\mathrm{p}} \exp \left(\int_{r_{\mathrm{sh}}}^{r} d r^{\prime} \frac{v\left(r^{\prime}\right)}{\kappa\left(r^{\prime}\right)}\right)} .
$$

Continuity mandates that $\omega_{\mathrm{pp}} / N_{\mathrm{p}} \propto \sqrt{v(r)}$. We can scale the density and the flow speed to their values at the thermal sub-shock, $N_{\mathrm{p} \text {,sh }}$ and $v_{\mathrm{sh}}$, and pull these quantities out of the integral.

The energy density in the magnetic turbulence likely increases toward the shock on account of turbulence driving, and so $(\delta B(r))^{2}$ falls off with increasing $r$. The cosmic-ray scattering rate is linked to the intensity of the turbulence, hence $\kappa(r)$ will rise with increasing $r$. We shall write the spatial profiles as

$$
(\delta B(r))^{2}=\left(\delta B_{\mathrm{sh}}\right)^{2} b(r) \quad \kappa(r)=\kappa_{\mathrm{sh}} k(r) .
$$

Note that $\delta B(r)$ may include small- $k$ turbulence that is driven by cosmic rays of energies higher than that of the particles whose energy losses we calculate. The diffusion coefficient increases with energy, and so does the precursor length, implying that at any given location in the precursor a particle sees turbulence that has been driven by cosmic rays of higher energy further out in the precursor.

We shall now transform the variable of integration in the argument of the exponential in eq. 12,

$$
x=\int_{r_{\mathrm{sh}}}^{r} d r^{\prime} \frac{v\left(r^{\prime}\right)}{\kappa\left(r^{\prime}\right)}
$$

which then reads

$$
\begin{aligned}
\dot{E}_{\mathrm{tot}} \lesssim & \frac{\omega_{\mathrm{pp}, \mathrm{sh}} N_{\mathrm{cr}, \mathrm{sh}}\left(\delta B_{\mathrm{sh}}\right)^{2} \kappa_{\mathrm{sh}}}{8 \pi \epsilon c N_{\mathrm{p}, \mathrm{sh}}} \\
& \times \int_{0}^{\infty} d x \sqrt{\frac{v(x)}{v_{\mathrm{sh}}}} b(x) k(x) \exp (-x),
\end{aligned}
$$

where $b(x)$ and $k(x)$ are defined in eq. 13 and eq. 14. In the quasilinear limit $\kappa \propto 1 /(\delta B)^{2}$ is approximately true, meaning $b(x) k(x) \approx$ const, whereas for Bohmian scaling one expects 
$b(x) k(x) \propto \sqrt{b(x)}$, falling off with increasing $x$. In the general case, $b(x) k(x)$ is at most constant, but more likely a declining function, and so the integral in eq. 15 yields a numerical factor close to unity.

The total energy-loss rate of cosmic rays in the precursor then is

$$
\dot{E}_{\mathrm{tot}} \lesssim U_{\delta B} \frac{N_{\mathrm{cr}, \mathrm{sh}}}{N_{\mathrm{p}}} \frac{\eta \Gamma_{\mathrm{cr}}}{3 \epsilon} \frac{c^{2}}{v_{\mathrm{A}}},
$$

likewise the spectral steepening,

$$
\Delta s=(s-1) \frac{1}{\frac{\dot{\mathrm{E}}_{\mathrm{acc}}}{\dot{E}_{\mathrm{tot}}}-1} \lesssim \frac{2(s-1)^{2} \eta M_{\mathrm{A}}}{3 \epsilon} \frac{U_{\delta B}}{U_{\mathrm{bulk}}} .
$$

\section{Summary and Discussion}

We calculated the energy-transfer rate from cosmic rays to non-resonant plasma waves in the precursor of the forward shock of an SNR. Two different ways of calculation led to essentially the same result for the softening of the particle spectra that is imposed by that energy transfer. It can be related to the energy-density ratio of amplified turbulent magnetic field and bulk plasma flow. That ratio is at most the inverse square of the Alfvénic Mach number of the thermal sub-shock, which we write with the full turbulent field amplitude,

$$
M_{\mathrm{A}}=\sqrt{\frac{U_{\mathrm{bulk}}}{U_{\delta B}+U_{B_{0}}}} .
$$

For turbulently amplified magnetic field, $U_{\delta B} \gg U_{B_{0}}$, the spectral steepening can hence be written either with the Alfvénic Mach number of the sub-shock or with the energy density of the turbulent field immediately upstream of the shock. The most important finding is then that for Bohm scaling of diffusion in the precursor, $\eta$, the change in spectral index is invariably

$$
\Delta s \lesssim \frac{2(s-1)^{2} \eta}{3 \epsilon M_{\mathrm{A}}}=\frac{2(s-1)^{2} \eta}{3 \epsilon} \sqrt{\frac{U_{\delta B}}{U_{\mathrm{bulk}}}} .
$$

Here $s$ is the cosmic-ray spectral index without steepening and $\epsilon$ is the magnetic fraction of the turbulent energy density immediately upstream of the shock. In Figure 1 we show this constraint as red exclusion area in a display of spectral softening vs. $M_{\mathrm{A}}$.

A consistency check is provided by mandating that in the steady state the wave-driving power per unit shock area (eq. 16) must be at least as large as the escape flux through the shock, $v_{\mathrm{sh}} U_{\delta B} / \epsilon$. This yields an implicit lower limit on the cosmic-ray density that can be used to calculate the number of exponential growth cycles available for Bell's mode [6]. We find at least one half exponential growth cycle for the nonresonant mode,

$$
U_{\mathrm{cr}} \geq \frac{6}{\eta} \frac{U_{\mathrm{bulk}}}{M_{\mathrm{A}}} \Rightarrow N_{\exp } \gtrsim 0.5 .
$$

Evidently that condition is met for any significant growth of the mode. As the mode has to grow from small fluctuations to a level $\delta B \gg B_{0}$, one would instead need at least ten exponential growth 


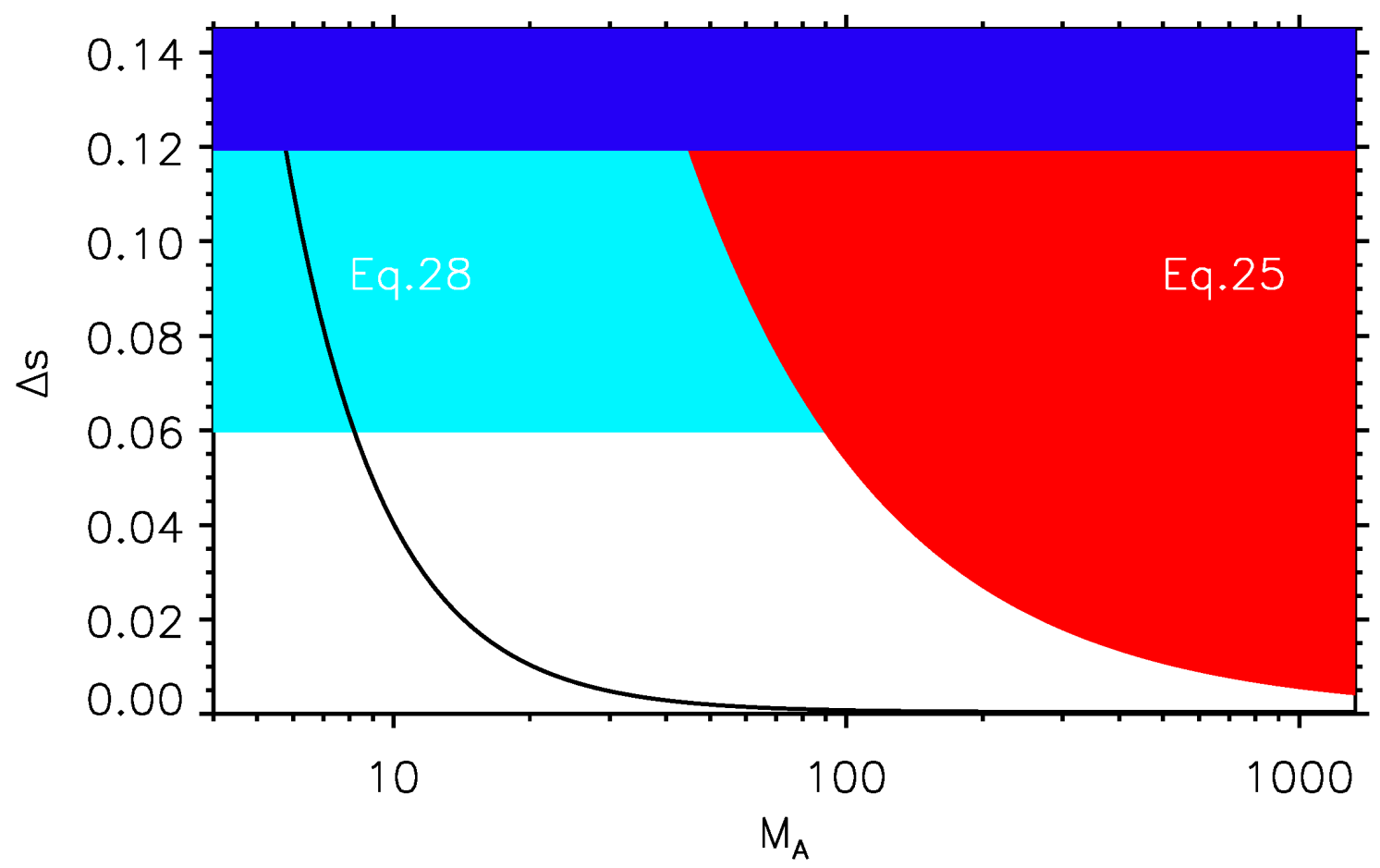

Figure 1: Exclusion limits for the spectral softening, $\Delta s$, as function of the Alfvénic Mach number, $M_{\mathrm{A}}$, assuming $\eta=4$ and $\epsilon=0.5$. The red area marks violation of eq. 19. The cyan and blue areas are excluded by eq. 22 for $v_{\mathrm{sh}} U_{\mathrm{cr}} / c U_{\text {bulk }}$ set to $10^{-3}$ and $4 \cdot 10^{-3}$, respectively.

cycles. Interestingly, we recover $\left.\Delta s\right|_{\text {Bell }} \approx 4 U_{\delta B} /\left(\epsilon U_{\text {cr }}\right)$, i.e. the result of Bell et al. [1], if we assume a cosmic-ray density that equals the right-hand side eq. 20 . In that case $N_{\exp } \simeq 0.5$, meaning there is no time for the mode to grow, and so the steady-state level $U_{\delta B} / U_{\mathrm{cr}} \approx v_{\mathrm{sh}} /(2 c)$ cannot be reached.

We calculated the level of spectral softening ignoring the condition $k r_{\mathrm{L}} \gg 1$ under which the non-resonant mode can be driven. This relation can for $\delta B \gg B_{0}$ be rephrased as

$$
\frac{v_{\mathrm{sh}}}{4 c} \frac{U_{\mathrm{cr}}}{U_{\delta B}} \gg 1 \text {. }
$$

This condition requires that the left-hand side be much larger than unity, but how much larger? Let us conservatively suppose that is it larger than or equal to two, and inserting that into eq. 19 we find

$$
\Delta s \lesssim \frac{(s-1) \eta}{3 \sqrt{2} \epsilon} \sqrt{\frac{v_{\mathrm{sh}}}{c} \frac{U_{\mathrm{cr}}}{U_{\text {bulk }}}} .
$$

This condition is also visible in Figure 1 for two rather high values of $v_{\mathrm{sh}}$ and $U_{\mathrm{cr}}$.

In conclusion, we find and show in Figure 1 that even for very efficient cosmic-ray acceleration, for which $\eta \approx 4$, and the highest magnetic-field amplification that is allowed for Bell's mode, the spectral softening appears to be moderate, $\Delta s \lesssim 0.1$, and it is negligible for standard SNR parameters. As we explicitely allow for spatial variation in the cosmic-ray precursor, the shock speed, $v_{\text {sh }}$, in Eq. 22 is that of the thermal subshock, not that in the far-upstream frame. This statement is based on the energy transfer that can be accomplished within the time available, and it does not assume that a certain saturation level of the wave energy density is reached. 


\section{References}

[1] Bell, A. R., Matthews, J. H., \& Blundell, K. M. 2019, MNRAS, 488, 2466.

[2] Bell, A. R. 2004, MNRAS, 353, 550.

[3] Brose, R., Pohl, M., Sushch, I., et al. 2020, A\&A, 634, A59.

[4] Drury, L. O. 1991, MNRAS, 251, 340.

[5] Giacalone, J. \& Jokipii, J. R. 2007, ApJL, 663, L41.

[6] Niemiec, J., Pohl, M., Stroman, T., et al. 2008, ApJ, 684, 1174.

[7] Ohira, Y., Murase, K., \& Yamazaki, R. 2010, A\&A, 513, A17.

[8] Reville, B. \& Bell, A. R. 2013, MNRAS, 430, 2873. 